\title{
ADVANCING ENVIRONMENTAL NOISE POLLUTION ANALYSIS IN URBAN AREAS BY CONSIDERING THE VARIATION OF POPULATION EXPOSURE IN SPACE AND TIME
}

\author{
S. Freire ${ }^{a, *}$, N. Gomes ${ }^{b}$ \\ a e-GEO, Geography and Regional Planning Research Centre, FCSH, Universidade Nova de Lisboa, Lisbon, Portugal - \\ sfreire@fcsh.unl.pt \\ ${ }^{\mathrm{b}} \mathrm{IN}+$, Center for Innovation, Technology and Policy Research, Instituto Superior Técnico, Oeiras, Portugal - \\ nuno.gomes@dem.ist.utl.pt
}

KEY WORDS: Environmental noise, Population exposure, Spatial analysis, Dasymetric mapping, Lisbon

\begin{abstract}
:
Ambient noise is a subtle form of pollution in large urban areas, degrading human health and well-being. In Europe, directives require that urban environmental noise be measured and mapped for the main periods of the daily cycle. Subsequent analyses of human exposure to noise in those periods is usually conducted using resident (i.e., nighttime) population from the census and assuming constant densities within the enumeration units. However, population distribution and densities vary considerably from night to day in metropolitan areas, and disregard for that process results in gross misestimation of exposure to ambient noise in the daytime period.

This study considers the spatio-temporal variation of population distribution in assessing exposure to ambient noise in a major urban area, the city of Lisbon, Portugal. Detailed and compatible day- and nighttime population distribution maps were used, developed by means of 'intelligent dasymetric mapping'. After categorizing noise levels in existing maps in each period, classified according to current legislation, human exposure to ambient noise was assessed with temporally matching population surfaces. Population exposure to noise in 2000 and 2009 was compared and further analyzed in regards to main source of noise, i.e. road traffic vs. aircraft.

Results show that human exposure to noise shifts substantially in time and space, with a significant increase in exposed population from the nighttime to daytime period, especially in the higher noise levels. This is due to the combined effects of the daily variation of noise patterns and population distribution.
\end{abstract}

\section{INTRODUCTION}

Ambient or community noise is an insidious and frequently overlooked form of pollution in large urban areas, having wellknown adverse effects in human health and well-being. For better understanding these effects, conducting accurate exposure assessment is critical (Gan et al., 2012).

In Europe, directives from the Commission (2002/49/EC) require that urban environmental noise be measured and mapped for the main sources and periods of the daily cycle. This is in order to capture the shifting nature of the phenomenon caused by changes in traffic and activity patterns. Further analysis of population exposure to noise in those distinct periods is usually conducted using resident (i.e., nighttime) population from the census and assuming constant densities within the enumeration units (e.g., Brainard et al., 2004). However, often population occupation of those units is not constant or exhaustive, and their densities vary considerably from night to day in metropolitan areas (Freire, 2010). Updated and detailed mapping of population distribution is important for decision support, if produced at appropriate spatial and temporal scales (Sutton et al., 2003). Disregard for these challenges may result in gross misestimation of exposure to ambient noise in the daytime period, while the spatial interpolation process adopted may cause additional inaccuracies.
Murphy et al. (2009) have estimated the exposure of residents and workers to traffic noise in day and night periods, using population data from the census. However, the analysis was only performed for a small area of Dublin, Ireland. Despite the recent advances, more research is needed on urban ambient noise exposure (Pujol et al., 2012).

Advancing to a city-wide comprehensive analysis of human exposure to noise requires that spatio-temporally detailed noise maps be combined with matching population distribution maps. When these latter data are not readily available from official censuses, they can be produced using geographic and cartographic modeling approaches, such as dasymetric mapping, i.e. using areal interpolation to combine best-available census data and mobility statistics with land use maps.

This work aims at improving the assessment of human exposure to ambient noise by introducing the spatio-temporal variation of population distribution in the analysis. Demonstration is conducted for the municipality of Lisbon, Portugal, for which detailed and compatible day- and nighttime population distribution maps were developed by means of 'intelligent' dasymetric mapping, i.e. using areal interpolation to combine best-available census data and mobility statistics with land use maps.

\footnotetext{
* Corresponding author.
} 
First, using existing maps, noise levels were reclassified into categories in each day and night period, according to current legislation. Then, human exposure to categories of ambient noise was assessed with temporally matching population surfaces. Exposed population was further analyzed in regards to main source of noise, i.e., road traffic vs. aircraft, and its evolution compared from 2000 to 2009.

\section{DATA AND STUDY AREA}

\subsection{Study Area}

The study area includes the whole municipality of Lisbon, the largest city and the capital of Portugal (Figure 1).

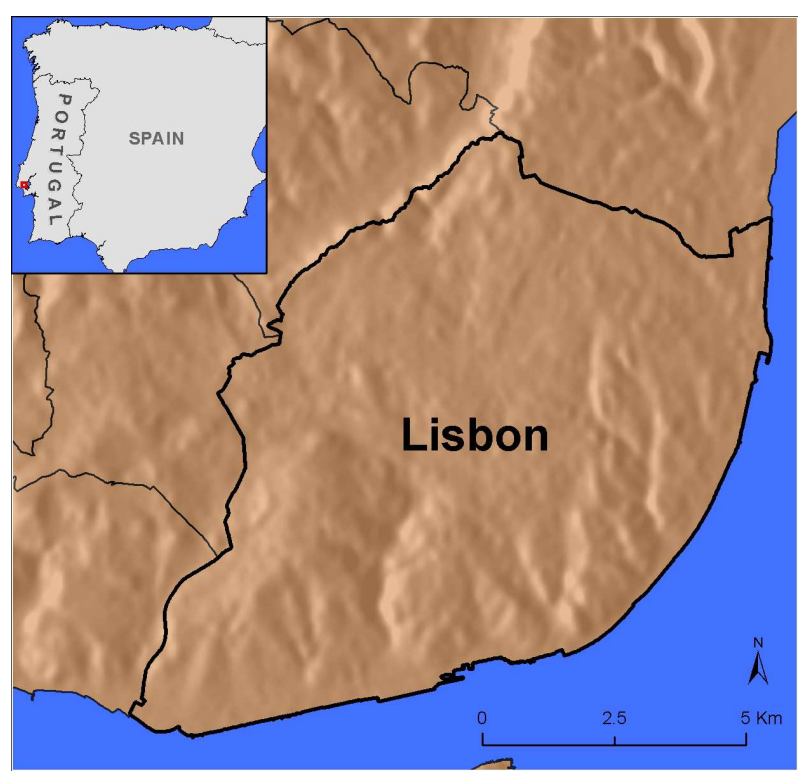

Figure 1. Study area - the municipality of Lisbon, Portugal

Lisbon occupies an area of $84 \mathrm{Km}^{2}$, and is a characteristic European capital city, with very diverse land cover/land use and urban morphologies. These range from historical neighborhoods (e.g., the downtown area of Baixa), where the street network is dense and most of the area is built-up, to modern residential areas (e.g., the area of Alta), with ongoing construction of roads and multi-family buildings.

Lisbon is home to about 550,000 residents, $5 \%$ of the country's population (INE, 2001). Although the city's average population density is around 6500 inhabitants per square kilometer, actual densities vary significantly in space and time. The city comprises areas with open land or scattered housing whose uneven population density is not well captured and represented by census polygons. These can be rather large even at the block level. Also, due to daily commuting for work and study purposes, the total daytime population of Lisbon differs by almost $60 \%$ of the residential figures from the census (INE, 2001; 2003). This process induces intense road traffic that causes sharp differences in noise levels between night and day.

The municipality experiences considerable levels of ambient noise, originating from different sources: the city is crossed by many multi-lane avenues and several freeways, and a major international airport is partially located within its limits, on the northern edge. However, such noise levels vary significantly in time and space.

The area is ideally suited for these analyses due to availability of adequate spatio-temporal population surfaces and ambient noise maps for different periods. Furthermore, the city of Lisbon is at the center of a larger metropolitan area with similarities to many other European urban centers.

\subsection{Data sets}

The main spatial datasets used in the course of the presented analyses were population distribution surfaces and ambient noise maps. These data are listed in Table 1.

\begin{tabular}{|l|l|l|}
\hline Data set & Date & Data type \\
\hline Global nighttime noise & 2000 & Vector polygon \\
\hline Global daytime noise & 2000 & Vector polygon \\
\hline Road traffic noise & 2000 & Vector polygon \\
\hline Aircraft noise & 2000 & Vector polygon \\
\hline Nighttime noise $\left(\mathrm{L}_{\mathrm{n}}\right)$ & 2009 & Vector polygon \\
\hline Daytime noise $\left(\mathrm{L}_{\mathrm{den}}\right)$ & 2009 & Vector polygon \\
\hline Nighttime population $(\mathrm{NP})$ & 2001 & Raster $(50 \mathrm{~m})$ \\
\hline Daytime population $(\mathrm{DP})$ & 2001 & Raster $(50 \mathrm{~m})$ \\
\hline $\begin{array}{l}\text { Daytime worker/student } \\
\text { population }(\mathrm{DWP})\end{array}$ & 2001 & Raster $(50 \mathrm{~m})$ \\
\hline $\begin{array}{l}\text { Daytime residential population } \\
\text { (DRP) }\end{array}$ & 2001 & Raster $(50 \mathrm{~m})$ \\
\hline
\end{tabular}

Table 1. Main input data sets used

Ambient noise maps for different periods and noise sources were obtained in vector format from the municipality of Lisbon. Four maps were available for the year 2000, representing noise levels for different periods and sources: global nighttime, global daytime, road traffic, and aircraft. These were mapped in 5 $\mathrm{dB}(\mathrm{A})$ increments, from $<=45$ to $>80$. Figure 2 shows the global nighttime noise map, whereas the global daytime map is presented in Figure 3.

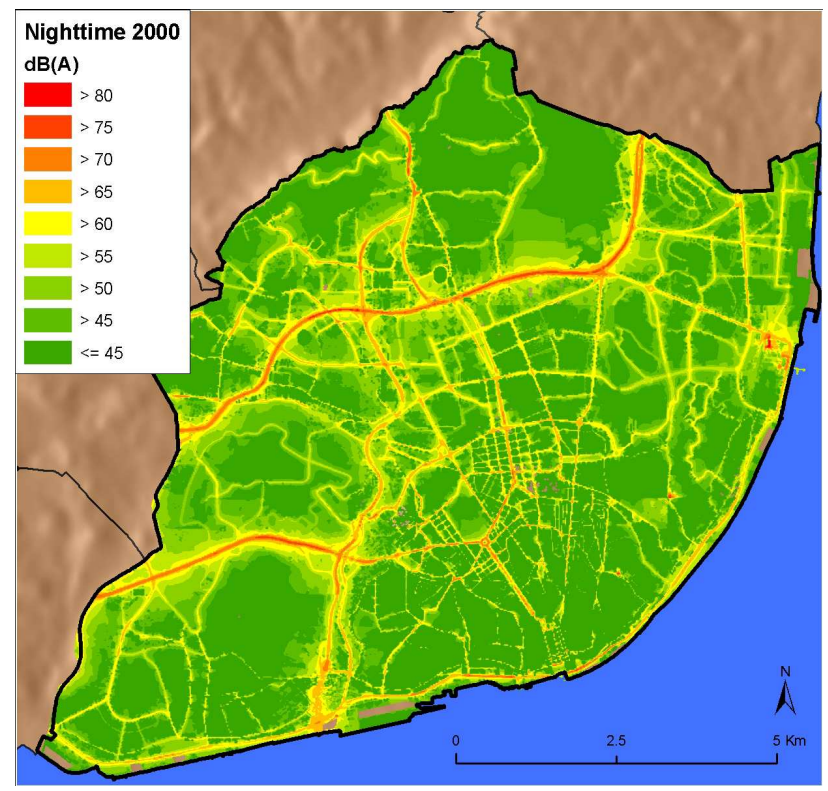

Figure 2. Global nighttime noise levels for Lisbon in 2000 


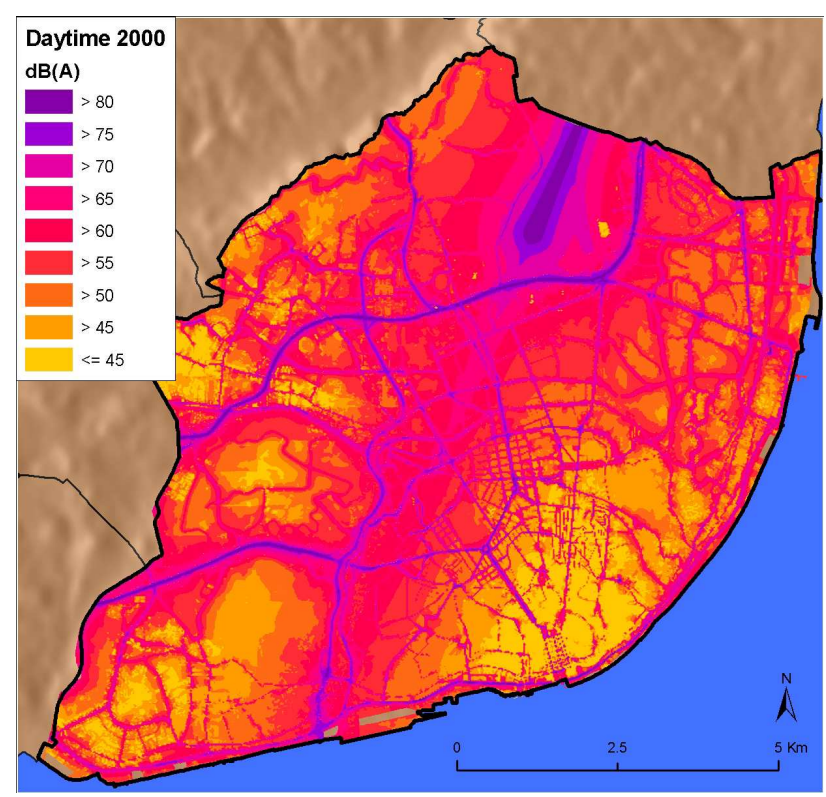

Figure 3. Global daytime noise levels for Lisbon in 2000

For 2009, only two noise layers were available, mapping noise levels using five classes: Nighttime noise $\left(\mathrm{L}_{\mathrm{n}}\right)$, and daytime noise $\left(\mathrm{L}_{\mathrm{den}}\right) . \mathrm{L}_{\mathrm{n}}$ represents the annual A-weighted long-term average sound pressure for the night period (23:00-7:00), while $\mathrm{L}_{\text {den }}$ refers to the entire daily cycle, hence including measurements taken in day, evening, and night periods (Figure $5)$.

Four population distribution surfaces were available for the study area: nighttime (residential) population (NP), daytime residential population (DRP), daytime worker and student population (DWP), and total daytime population (DP). These surfaces were developed previously by the first author as $50-\mathrm{m}$ resolution raster grids for the Lisbon Metropolitan Area, based on data from 2001. These grids represent maximum expected densities on a typical workday assuming that everyone is at home at night and all workers and students are in their workplaces and schools, and the remainder is in their residences during the daytime period. Although this is still a simplification of reality, it is a major improvement over existing data sets that can benefit analyses from regional to local scale.

\section{METHODOLOGY}

\subsection{Assessing Population Exposure to Ambient Noise in 2000}

Population exposure to ambient noise in Lisbon was assessed in GIS using zonal analysis to summarize the modeled population surfaces with the temporally matching noise map. It was assured that both datasets were in the same projected coordinate system.

The modeling of population distribution for the LMA is based on raster dasymetric mapping and areal interpolation using street centerlines as spatial reference units to re-allocate population counts. The model considers land use and population mobility statistics (INE, 2003), combining 'intelligent dasymetric mapping' (Mennis and Hultgren, 2006) with the approach proposed by McPherson and Brown (2004). More details about the development of these population surfaces are available in Freire and Aubrecht, 2012, and Freire et al., 2013.

The main goal of this study was to analyze and compare noise exposure in nighttime and daytime periods with temporally matching population surfaces. However, for legal reasons, nighttime $\left(\mathrm{L}_{\mathrm{n}}\right)$ and daytime $\left(\mathrm{L}_{\mathrm{den}}\right)$ noise reference thresholds are different, and so are the levels used in map legends. Therefore it was necessary to convert both quantitative noise level classifications in each period (in $\mathrm{dB}(\mathrm{A})$ to a common qualitative scale. This conversion is shown in Table 2.

\begin{tabular}{|l|l|l|}
\hline Level $\mathbf{L}_{\mathbf{n}}$ & Level $\mathbf{L}_{\mathbf{d e n}}$ & Category \\
\hline$<=45$ & $<=55$ & Low \\
\hline$>45-50$ & $>55-60$ & Moderate \\
\hline$>50-55$ & $>60-65$ & High \\
\hline$>55-60$ & $>65-70$ & Very High \\
\hline$>60$ & $>70$ & Extreme \\
\hline
\end{tabular}

Table 2. Reclassification of noise levels into categories

The five quantitative noise classes were converted to five ordinal categories, from 'Low' to 'Extreme'. These categories were defined considering current legislation concerning noise effects and tolerable thresholds, and were used to summarize the number of exposed people.

Then, in order to fully assess the noise exposure situation in 2000, four analyses were implemented, using the four noise maps available: (1) exposure of nighttime and daytime population to global nighttime and daytime noise (2) exposure of daytime population DWP and DRP to global daytime noise, (3) exposure of daytime population DWP and DRP to road traffic noise, and (4) exposure of daytime population DWP and DRP to aircraft noise. In each of these analyses, potentially affected people were quantified and differences analyzed.

\subsection{Comparing Population Exposure to Noise 2000 - 2009}

A second analysis aims at comparing human exposure to noise between 2000 and 2009, in nighttime and daytime periods. The analysis mainly assesses changes in noise patterns between maps for these dates, since population distribution is assumed to remain constant - the only available population surfaces are the ones for 2001. However, the daytime map for 2009 uses $L_{d e n}$ as noise indicator, which incorporates measurements acquired in day-evening-night (despite representing mostly a worst-case scenario occurring in the daytime period). For this reason, we decided to also produce an averaged day-night population grid in order to best match the 2009 noise map.

The average population (AP) surface was derived from the nighttime population (NP) and daytime population (DP) grids and computed for each cell in the study area using the following map algebra expression:

$$
\mathrm{AP}=(\mathrm{NP}+\mathrm{DP}) / 2
$$

This new surface represents a 24-h averaged population density for a typical workday.

Figures 4 and 5 combine the population distribution and noise maps over satellite imagery, using background from Google 
Earth. Population density is illustrated in 3-D, with the height of each 50 -m cell being proportional to its number of persons.

Figure 4 shows nighttime population (NP) densities overlain on the Nighttime noise $\left(\mathrm{L}_{\mathrm{n}}\right)$ categories in 2009; Figure 5 shows averaged population (AP) densities overlain on daytime noise $\left(\mathrm{L}_{\mathrm{den}}\right)$ categories in 2009.

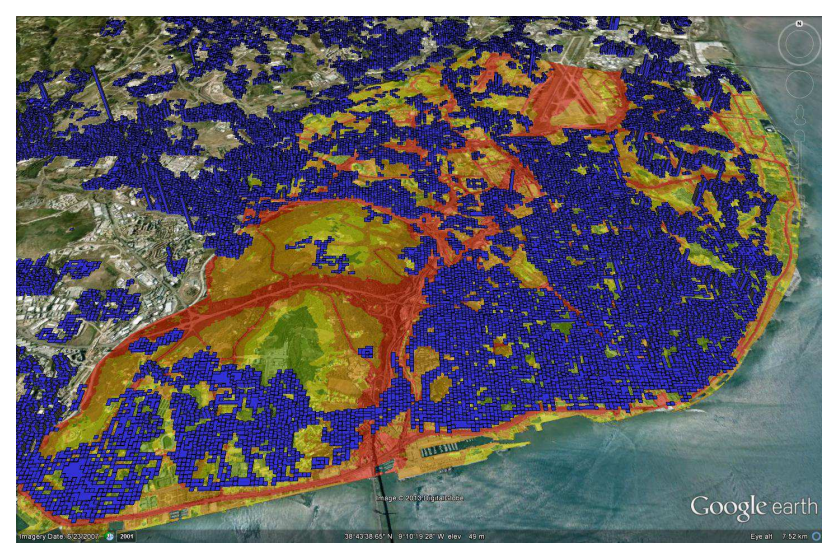

Figure 4. Nighttime noise $\left(\mathrm{L}_{\mathrm{n}}\right)$ categories in 2009 and nighttime population (NP) density for Lisbon

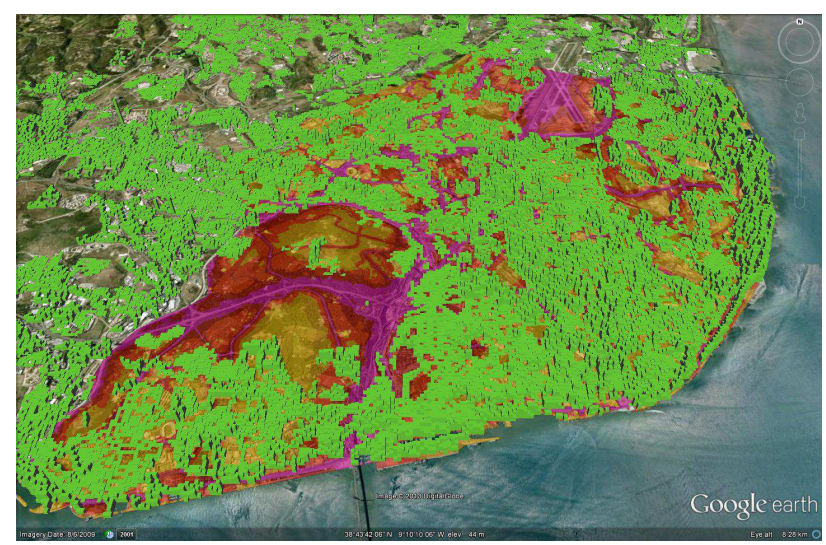

Figure 5. Daytime noise $\left(\mathrm{L}_{\mathrm{den}}\right)$ categories in 2009 and averaged population (AP) density for Lisbon

Comparing both figures, it can be seen that in Figure 5 zones of higher noise categories are larger and that averaged night-day population distribution is more widespread in the city than the residential distribution, occupying new areas.

\section{RESULTS AND DISCUSSION}

\subsection{Assessing Population Exposure to Ambient Noise in 2000}

Results of the analysis of human exposure to noise maps in the year 2000 in Lisbon are presented in Tables 3 to 6.

Table 3 shows striking differences from nighttime to daytime, with exposure rising in all noise categories except in the 'Low' class; it increases by as much as $200 \%$ and 200,000 people in the 'High' and 'Very High' categories. While at night the 'Low' noise category includes $63 \%$ of the residents, it affects only $34 \%$ of the daytime population.

\begin{tabular}{|l|r|r|r|r|r|r|}
\hline \multirow{2}{*}{$\begin{array}{l}\text { Noise } \\
\text { categ. }\end{array}$} & \multicolumn{2}{|l|}{ Night } & \multicolumn{2}{l|}{ Day } & \multicolumn{2}{l|}{ Difference } \\
\cline { 2 - 7 } & Persons & \multicolumn{1}{l|}{$\%$} & Persons & \% & Persons & $\%$ \\
\hline Low & 353278 & 62,9 & 305363 & 34,3 & -47915 & $-13,6$ \\
\hline Moderate & 59032 & 10,5 & 163415 & 18,3 & 104384 & 176,8 \\
\hline High & 54155 & 9,6 & 164063 & 18,4 & 109907 & 202,9 \\
\hline V. High & 45122 & 8,0 & 135463 & 15,2 & 90341 & 200,2 \\
\hline Extreme & 49989 & 8,9 & 122824 & 13,8 & 72835 & 145,7 \\
\hline Total & 561575 & 100 & 891128 & 100 & 329553 & 58,7 \\
\hline
\end{tabular}

Table 3. Nighttime vs. daytime population exposure to noise in Lisbon in 2000

\begin{tabular}{|l|r|r|r|r|}
\hline \multirow{2}{*}{ Noise categories } & \multicolumn{2}{l|}{ DWP } & \multicolumn{1}{l|}{ DRP } \\
\cline { 2 - 5 } & \multicolumn{1}{l|}{ Persons } & \multicolumn{1}{l|}{$\%$} & Persons & \multicolumn{1}{l|}{} \\
\hline Low & 167612 & 28,5 & 137751 & 45,4 \\
\hline Moderate & 105676 & 18,0 & 57740 & 19,0 \\
\hline High & 111336 & 19,0 & 52727 & 17,4 \\
\hline V. High & 103686 & 17,7 & 31778 & 10,5 \\
\hline Extreme & 99140 & 16,9 & 23684 & 7,8 \\
\hline Total & 587449 & 100 & 303679 & 100 \\
\hline
\end{tabular}

Table 4. Daytime worker (DWP) and residential (DRP) exposure to daytime noise in Lisbon in 2000

Looking in more detail at daytime exposure in 2000 (Table 4), it can be observed that most of the population is affected by 'Moderate' to 'Extreme' levels, but the problem is much more acute for the worker/student population. $34 \%$ of this population is located in 'V. High' and 'Extreme' noise zones, compared to $18 \%$ of the daytime residents. The map of road traffic noise represents mostly a worst-case scenario occurring in daytime period.

\begin{tabular}{|l|r|r|r|r|r|r|}
\hline \multirow{2}{*}{$\begin{array}{c}\text { Noise } \\
\text { categories }\end{array}$} & \multicolumn{2}{|c|}{ DWP } & \multicolumn{2}{c|}{ DRP } & \multicolumn{2}{c|}{ DP } \\
\cline { 2 - 7 } & Persons & \% & Persons & \% & Persons & \% \\
\hline Low & 270509 & 46,2 & 199641 & 65,9 & 470150 & 52,9 \\
\hline Moderate & 82467 & 14,1 & 31760 & 10,5 & 114227 & 12,9 \\
\hline High & 70210 & 12,0 & 23564 & 7,8 & 93774 & 10,6 \\
\hline V. High & 77400 & 13,2 & 27084 & 8,9 & 104484 & 11,8 \\
\hline Extreme & 84850 & 14,5 & 20970 & 6,9 & 105820 & 11,9 \\
\hline Total & 585436 & 100 & 303019 & 100 & 888455 & 100 \\
\hline
\end{tabular}

Table 5. Exposure of daytime worker (DWP), residential (DRP), and daytime total (DP) to road traffic noise in Lisbon in 2000

Concerning exposure to road traffic noise, Table 5 reveals that while most of the daytime population is in the 'Low' category, this is mostly due to the residential component. In fact, exposure to higher noise levels is more significant for 
worker/student populations, $18 \%$ of which are located in 'V. High' and 'Extreme' noise zones.

Regarding aircraft noise exposure, Table 6 shows that this source is less problematic than noise originating in road traffic. By large most of the daytime population is in the 'Low' category, with similar rates of exposure for DWP and DRP populations decreasing with increase in noise categories.

However, still about 3600 workers may be affected by 'Extreme' noise levels. Only daytime population distribution is considered since operations at the Lisbon international airport in nighttime period are very limited.

\begin{tabular}{|l|r|r|r|r|r|r|}
\hline \multirow{2}{*}{$\begin{array}{c}\text { Noise } \\
\text { categories }\end{array}$} & \multicolumn{2}{|c|}{ DWP } & \multicolumn{2}{c|}{ DRP } & \multicolumn{2}{c|}{ DP } \\
\cline { 2 - 7 } & Persons & \% & Persons & \% & Persons & $\%$ \\
\hline Low & 420626 & 71,6 & 228804 & 75,3 & 649430 & 72,9 \\
\hline Moderate & 91859 & 15,6 & 45753 & 15,1 & 137612 & 15,4 \\
\hline High & 48406 & 8,2 & 24405 & 8,0 & 72810 & 8,2 \\
\hline V. High & 22937 & 3,9 & 4320 & 1,4 & 27257 & 3,1 \\
\hline Extreme & 3622 & 0,6 & 397 & 0,1 & 4020 & 0,5 \\
\hline Total & 587449 & 100 & 303679 & 100 & 891128 & 100 \\
\hline
\end{tabular}

Table 6. Exposure of daytime worker (DWP), residential (DRP), and daytime total (DP) to aircraft noise in Lisbon in 2000

In general, results for 2000 show that exposure to noise considerably increases from nighttime to daytime, especially due to rising road traffic noise. 'High' and 'Very High' noise categories affect a very significant share of the daytime population, in particular the larger worker/student population present in the city.

\subsection{Comparing Population Exposure to Noise 2000 - 2009}

Concerning the analysis of changes in exposed population to noise from 2000 to 2009, results are presented in Tables $7-8$. The analyses reveal significant changes in the evolution of exposure from 2000 to 2009, although with specific differences between the nighttime and the daytime periods.

\begin{tabular}{|l|r|r|r|r|r|r|}
\hline \multirow{2}{*}{$\begin{array}{c}\text { Noise } \\
\text { categories }\end{array}$} & \multicolumn{2}{|c|}{$\mathbf{2 0 0 0}$} & \multicolumn{2}{c|}{$\mathbf{2 0 0 9}$} & \multicolumn{2}{c|}{ Difference } \\
\cline { 2 - 7 } & Persons & \multicolumn{1}{c|}{$\%$} & Persons & \% & Persons & $\%$ \\
\hline Low & 353278 & 62,9 & 222410 & 39,5 & -130867 & $-37,0$ \\
\hline Moderate & 59032 & 10,5 & 94443 & 16,8 & 35412 & 60,0 \\
\hline High & 54155 & 9,6 & 80416 & 14,3 & 26261 & 48,5 \\
\hline V. High & 45122 & 8,0 & 79657 & 14,2 & 34535 & 76,5 \\
\hline Extreme & 49989 & 8,9 & 85828 & 15,3 & 35839 & 71,7 \\
\hline Total & 561575 & 100 & 562755 & 100 & 1180 & 0,21 \\
\hline
\end{tabular}

Table 7. Nighttime population exposure to noise in Lisbon in 2000 vs. 2009

Table 7 shows that exposure increases in all noise categories except in the 'Low' class; it rises by more than $70 \%$ and 70,000 people in the 'Very High' and 'Extreme' categories. While in 2000 the 'Low' noise category includes $63 \%$ of the nighttime residents, in 2009 it includes only $40 \%$ of the same residents, with the remainder $60 \%$ being affected by higher noise levels. (Differences in total number of persons are caused by the 2000 map having 'holes' where noise data is absent.)

\begin{tabular}{|l|r|c|c|c|r|r|}
\hline \multirow{2}{*}{$\begin{array}{c}\text { Noise } \\
\text { categories }\end{array}$} & \multicolumn{2}{|c|}{$\mathbf{2 0 0 0}$} & \multicolumn{2}{c|}{$\mathbf{2 0 0 9}$} & \multicolumn{2}{c|}{ Difference } \\
\cline { 2 - 7 } & Persons & \% & Persons & \% & Persons & \% \\
\hline Low & 280296 & 38,6 & 268241 & 36,9 & -12054 & $-4,3$ \\
\hline Moderate & 135202 & 18,6 & 114840 & 15,8 & -20362 & $-15,1$ \\
\hline High & 130898 & 18,0 & 107068 & 14,7 & -23829 & $-18,2$ \\
\hline V. High & 97187 & 13,4 & 110083 & 15,1 & 12896 & 13,3 \\
\hline Extreme & 83360 & 11,5 & 126710 & 17,4 & 43350 & 52,0 \\
\hline Total & 726941 & 100 & 726941 & 100 & 0 & 0,0 \\
\hline
\end{tabular}

Table 8. Daytime population exposure to noise in Lisbon in 2000 vs. 2009

Regarding average day-night population exposure to daytime noise, Table 8 shows a different situation. While rates of exposure to specific categories are relatively similar, exposure to 'Extreme' noise levels appears to increase dramatically, by $52 \%$ (additional 43,000 persons exposed).

It should be noted that although changes in exposure from 2000 to 2009 are significant, these differences are only attributable to shifts in noise patterns and/or noise measurements, since the population distribution surfaces are the same. If these surfaces could be updated to represent the latter date, presumably these figures would be different.

\section{CONCLUSIONS}

The present work is an initial approach towards considering the spatio-temporal variation of population distribution in the analysis of exposure to ambient noise in a major urban area. Several time-specific detailed surfaces of population were combined with different ambient noise layers to estimate human exposure to specific noise categories. More relevant than the specific population figures, is the demonstration that in large cities human exposure to harmful environmental conditions varies significantly in time and space.

Results show that, in Lisbon, human exposure to noise shifts substantially in time and space, with many more people exposed to higher noise levels in the daytime period, especially due to rising road traffic noise. 'High' and 'Very High' noise categories affect a very significant share of the daytime population, in particular the larger worker/student population present in the city. From 2000 to 2009, exposure increases in the two highest, most damaging noise categories, especially in the nighttime period. This is due to the combined effects of the daily variation of noise patterns and population distribution, caused by changes in human activities. Geographic information and analysis should account for these shifts and be compatible both in time and space. 
Such a city-wide assessment can provide valuable information for implementation of effective noise mitigation measures, not yet adopted in the municipality.

Planned future developments include conducting a more detailed modeling of population distribution in space (preferably at the building level considering its use and human occupation) and also for different time periods (e.g., rush hour, week-ends). Updating population surfaces with data from Census 2011 will be possible when mobility statistics become available.

\section{REFERENCES}

Brainard, S., Jones, A., Bateman, I., Lovett, A., 2004. Exposure to Environmental Urban Noise Pollution in Birmingham. $U K$. Urban Studies, 41 (13), pp. 2581-2600.

Freire, S., 2010. Modeling of spatio-temporal distribution of urban population at high-resolution - value for risk assessment and emergency management. In: Konecny, M., Zlatanova, S., Bandrova, T.L. (eds.), Geographic Information and Cartography for Risk and Crisis Management. Lecture Notes in Geoinformation and Cartography. Springer Berlin Heidelberg, pp. 53-67.

Freire, S., Aubrecht, C., 2012. Integrating population dynamics into mapping human exposure to seismic hazard. Natural Hazards \& Earth Systems Sciences, 12 (11), pp. 3533-3543.

Freire, S., Aubrecht, C., Wegscheider, S., 2013. Advancing tsunami risk assessment by improving spatio-temporal population exposure and evacuation modeling. Natural Hazards, pp. 1-14.

Gan W., McLean, K., Brauer, M., Chiarello, S., Davies, H., 2012. Modeling Population Exposure to Community Noise and Air Pollution in a Large Metropolitan Area. Environmental Research, pp. 11-16.

INE (Instituto Nacional de Estatística), 2001. Recenseamento Geral da População e da Habitação, Lisbon.

INE (Instituto Nacional de Estatística), 2003. Movimentos Pendulares e Organização do Território Metropolitano: Área Metropolitana de Lisboa e Área Metropolitana do Porto 19912001, Lisbon.

McPherson, T., Brown, M., 2003. Estimating daytime and nighttime population distributions in U.S. cities for emergency response activities. 84th AMS Annual Meeting, pp. 1-10

Mennis, J. Hultgren, T., 2006. Intelligent dasymetric mapping and its application to areal interpolation. Cartography and Geographic Information Science, 33(3), pp. 179-194.

Murphy, E., King, E., Rice, H., 2009. Estimating Human Exposure to Transport Noise in Central Dublin, Ireland. Environment International, 35 (2), pp. 298-302.

Pujol, S., Berthillier, M., Defrance, J., Lardiès J., Petit, R., Houot, H., Levain, J., Masselot, C., Mauny, F., 2012. Urban Ambient Outdoor and Indoor Noise Exposure at Home: A Population-based Study on Schoolchildren. Applied Acoustics, 73 (8), pp. 741-750.
Sutton, P., Elvidge, C., Obremski, T., 2003. Building and evaluating models to estimate ambient population density. Photogrammetric Engineering \& Remote Sensing, 69(5), pp. 545-553. 